\title{
Analytic and algebraic ideas : how to profit from their complementarity
}

\author{
Alain Yger \\ LaBAG \\ Université Bordeaux 1 \\ 351 Cours de la Libération \\ 33405 Talence \\ France yger@math.u-bordeaux.fr and labag@math.u-bordeaux.fr
}

To my friend Carlos on the occasion of his 60th birthday.

Summary. Facing algebraic questions from the analytic point of view has been the guide line of the joint work which I have been pursuing for almost twenty years with Carlos Berenstein. Instead of giving an up-to-date state of the art , I will focus on a few key points which still remain to be clarified and indicate a list of prospective developments where the ideas that analysis suggests, combined with multidimensional residue theory, will certainly have to play a major role. I will point out the following crucial fact, namely that in the "dictionary" between the analytic and algebraic points of view in multidimensional residue theory, integral symbols happen to be the analytic substitutes for power series developments in terms of parameters. Briançon-Skoda theorem, which seems to be a corner stone between constructions inspired by algebraic ideas on one side and by analytic ideas on the other side, will be a leitmotiv in this talk. Such ideas need to be combined in the future with arithmetic aspects we missed up to now and which indeed imply some additional rigidity.

\section{Introduction}

The concept of Chow ideal, together with the algebraic notion of integral closure and Briançon-Skoda theorems (that I will present in section 2), played a crucial role in D.W. Brownawell's approach to the effective nullstellensatz [Bro]. Later on, J. Kollár proposed in [Ko1] an alternative way to attack this problem which was more directly inspired by algebraic geometry. The search for a sharp arithmetic nullstellensatz (which was finally obtained by T. Krick, L.M. Pardo, M. Sombra in [KPS]) was our main motivation with Carlos Berenstein to re-interpret in the setting of multidimensional residue theory integral representation formulas ([BeY4]). Here comes the following crucial fact : in 
the "dictionary" between the analytic point of view (dealing for example with integral representation formulas of the Bochner-Martinelli type as in [BeY2]) and the algebraic point of view (inspired by multidimensional residue theory, as in [BeY4]), integral symbols happen to be the analytic substitutes for power series developments in terms of parameters. As an illustration, I will focus in section 2 on different approaches of Briançon-Skoda theorem which seems to be a corner stone in our constructions lying in between analysis and commutative algebra.

The notion of integral closure is also deeply connected with analytic approaches toward intersection theory. For example, given an intersection cycle $C=C_{1} \bullet \cdots \bullet C_{M}$, Lelong numbers related to $C$ or Lojasiewicz exponents for $I(C)$ (see $[\mathrm{CyKT}]$ ) are connected to the Chow ideal of $C$ and therefore to the integral closure of the ideal generated by the ideals corresponding to the cycles involved in the intersection. Residual currents constructed from Bochner-Martinelli representation formulas appear to play a crucial role in a generalization to improper intersections of the Lelong-Poincaré factorization formula

$$
\left[V\left(f_{1}, \ldots, f_{m}\right)\right]=\left(\bigwedge_{j=1}^{m} \bar{\partial} \frac{1}{f_{j}}\right) \wedge d f_{1} \wedge \cdots \wedge d f_{m} .
$$

The relation with King's formula is now better understood since the recent work of M. Méo [Meo1, Meo2] and M. Andersson [And3] (following [PTY] and [BeY5]). We will point out in section 2 how such results could be related to improper intersection theory, as introduced in $[\mathrm{Tw}]$.

I will conclude with an invitation to pursue such a program, taking more into account some arithmetic aspects it seems we missed up to now. Note for example that understanding Briançon-Skoda theorem from the algebraic point of view is much easier in positive characteristic, or that the theory of exponential polynomials (which initially inspired our joint work with Carlos Berenstein) has been recently revisited starting with $p$-adic ideas introduced by B. Dwork, N. Katz, P. Robba and more recently by Y. André ([DGS, Andr]). For example, Ritt's factorization theorem (which plays, together with analytic division formulas and Ax-Schanuel theorem, a fundamental role in our study of ideals generated by exponential-polynomials [BeY1]) is more rigid in the arithmetic context : in particular, (see [Andr]), the quotient by $z-1$ of a sum of exponential

$$
\sum_{j} a_{j} \exp \left(i \alpha_{j} z\right)
$$

(with $a_{j}, \alpha_{j} \in \overline{\mathbb{Q}}$ ) which vanishes at $z=1$ happens to be a sum of exponentials, which of course fails without arithmetic constraints (this leads to an elegant proof of Lindemann-Weierstrass theorem). There seems to me to be a parallel between the power of reasoning in positive characteristic when understanding in some algebraic way such mysterious division results as Briançon-Skoda's theorem and that of using ideas issued from $p$-adic analysis to get such rigid- 
ity results for exponential polynomials with both algebraic coefficients and frequencies.

\section{Chow ideal, integral closure and Briançon-Skoda theorem}

Let us adopt here a geometric (semi-local) point of view. Let $U$ be a neighborhood of the origin in $\mathbb{C}^{n}$ and $C=\sum_{j=1}^{N} \alpha_{j} Z_{j}, \alpha_{j} \in \mathbb{N}^{*}$, be a purely $k$-dimensional effective cycle with support $|C|$ in $U$. The Chow ideal of the cycle is defined as follows (see [Ko2]) : consider all admissible linear projections $\pi: \mathbb{C}^{n} \longrightarrow \mathbb{C}^{k+1}$ such that the origin is isolated in $\operatorname{Ker} \pi \cap|C|$ and the restriction of $\pi$ to $U \cap|C|$ is proper (in the topological sense) ; for such a map, let $\mu_{\pi, j}, j=1, \ldots, N$, be the number of sheets of the covering $\pi_{U \cap\left|Z_{j}\right|}: U \cap\left|Z_{j}\right| \longrightarrow \pi(U)$ and $F_{\pi}:=\prod_{j}\left(f_{\pi, j} \circ \pi\right)^{\alpha_{j} \mu_{\pi, j}}$, where $f_{\pi, j}=0$ is an irreducible equation for the hypersurface $\pi\left(\left|Z_{j}\right| \cap U\right)$ in $\pi(U)$; the Chow ideal $I^{\text {chow }}(C)$ of the effective cycle $C$ is defined as the ideal generated by the $F_{\pi}, \pi$ being such an admissible projection. Such a definition can be extended to non-purely dimensional cycles $\left(I^{\text {chow }}\left(C_{1}+C_{2}\right)=I^{\text {chow }}\left(C_{1}\right) \cdot I^{\text {chow }}\left(C_{2}\right)\right)$. A key fact relating intersection problems (of geometric nature) and division problems (of algebraic nature) is the following : if $C_{1}, \ldots, C_{M}$ are $M$ purely dimensional effective cycles with support in $U$ and $C_{1} \bullet \cdots \bullet C_{M}$ denotes the intersection cycle obtained through the Vogel-Tworzewski construction [Tw], then one has

$$
I^{\text {chow }}\left(C_{1} \bullet C_{2} \bullet \cdots \bullet C_{M}\right) \subset \overline{\left(I\left(C_{1}\right), \ldots, I\left(C_{M}\right)\right)},
$$

where the ideal of an effective cycle $C=\sum_{j} a_{j} Z_{j}$ is defined as

$$
I(C):=\prod_{j}\left(\left\{f ; f=0 \text { on }\left|Z_{j}\right|\right\}^{\alpha_{j}}\right.
$$

and the bar over the right-hand side of this inclusion means the integral closure of the ideal. We recall here that the integral closure of an ideal $I$ in a noetherian domain $R$ is defined as the ideal $\bar{I}$ of all elements in $R$ which satisfy an homogeneous relation of integral dependency

$$
h^{N}+a_{1} h^{N-1}+\cdots+a_{N} \equiv 0
$$

with $a_{k} \in I^{k}$ for $k=1, \ldots, N$. Equivalently, $\bar{I}$ consists of all elements $h$ such that $h \in I V$ for all discrete valuation rings $V$ lying between $R$ and its fraction field (3'), or all elements $h \in R$ such that there exists $c \in R^{*}$ with $c h^{n} \in I^{n}$ for infinitely many $n \in \mathbb{N}(3$ ") (see for example [Smi] for a survey and an updated list of references about such a notion, together with its relation to the notion of tight closure in a noetherian domain with prime characteristic on which we will come back later on). 
Summarizing here, we have at least three equivalent ways to assert that some $h \in R$ belongs to the integral closure of $I$. In the particular case $R=\mathcal{O}_{n}$, testing the valuative criterion for $h$ amounts to say that, given a system $\left(f_{1}, \ldots, f_{m}\right)$ of generators for $I$, one has $|h| \leq K \max \left|f_{j}\right|$ for some constant $K$ in a neighborhood of the origin. Such an analytic characterization will be deeply connected with the use of integral formulas of the Bochner-Martinelli type in order to solve explicitly division problems : generalized versions of the Lelong-Poincaré formulas expressed in terms of such integral formulas lead (when variables are duplicated) to extensions of Lagrange interpolation formulas of the Cauchy-Weil-Bergman type [We]. It remains an interesting challenge, in the case $R=\mathcal{O}_{n}$, to show (without going through resolution of singularities and the valuative criterion as in [LejT]) that any $h$ satisfying an inequality $|h| \leq K \max \left|f_{j}\right|$ fulfills an algebraic identity of the form (3).

When $R$ is a regular $n$-dimensional local ring (such as $\mathcal{O}_{n}$ ), then a fundamental result (initially proved in $\mathcal{O}_{n}$ by J. Briançon and H. Skoda in [BriS] using $L^{2}$-methods, then extended by J. Lipman and A. Sathaye in the general setting $[\mathrm{LiS}]$ ) asserts that for any ideal $I$ in $R$, for any $k \in \mathbb{N}^{*}$, the integral closure of $I^{k+\mu-1}$ lies in $I^{k}$ (in particular $\overline{I^{\mu}} \subset I$ taking $k=1$ ) if $\mu$ is defined as the minimum of $n$ and of the least number of generators of $I$. When $R$ has positive characteristic $p$, the proof of this result (for $k=1$ ) follows easily from the rather immediate intermediate inclusions $\overline{I^{r}} \subset I^{*} \subset I$, where $r$ denotes the minimal number of generators of $I$ and $I^{*}$ denotes the tight closure of $I=\left(f_{1}, \ldots, f_{r}\right)$, that is the set of $h \in R$ such there exists a nonzero element $c \in R$ with $c h^{p^{e}} \in\left(f_{1}^{p^{e}}, \ldots, f_{r}^{p^{e}}\right)$ for all $e>>0$. One needs here to use the last equivalent formulation (3") we proposed above for the membership to the integral closure [HoH1, Smi]. In the 0-characteristic case, such arguments do not apply directly, but it is fundamental to notice (as pointed out by B. Teissier in [Te3]), that Caratheodory theorem combines with a generalization of a theorem of Fenchel [HanR] to provide a simple proof of Briançon-Skoda theorem for monomial ideals. One should add here that the descent from characteristic $p$ down to characteristic zero, which was very recently described in [HoH2], provides indeed some new light on Briançon-Skoda theorem. It seems important to connect the naïve ideas we discuss here (inspired by BochnerMartinelli type integral formulas or by their algebraic counterpart, multidimensional residue theory in its algebraic presentation, for example [Lip]), to such an approach going from characteristic $p$ down to characteristic 0 .

Chow ideas (initially developed by Y. Nesterenko) were used in 1987 by D.W. Brownawell who combined them with Briançon-Skoda theorem in order to state the following striking result :

Theorem ([Bro]) Let $P_{1}, \ldots, P_{m}$ be $m$ polynomials in $\mathbb{C}\left[X_{1}, \ldots, X_{n}\right]$ such that $\operatorname{deg} P_{j} \leq D, j=1, \ldots, m$, with no common zeroes in $\mathbb{C}^{n} ;$ then one can write a Bézout identity $1=P_{1} Q_{1}+\cdots+P_{m} Q_{m}$ such that $\max \operatorname{deg} P_{j} Q_{j} \leq 3 n \mu D^{\mu}$, where $\mu:=\min (n, m)$. 
As a matter of fact, Brownawell's result comes as a combination of Bézout theorem, the inclusion (1), and finally Briançon-Skoda theorem which plays the role of a "black box". The following result (which is due to M. Hickel and answers positively a question our analytic point of view with Carlos Berenstein motivated us to ask in [BeY3]) emphasizes the crucial role of Briançon-Skoda theorem as a path from intersection to division problems (see also [EL] for a related point of view) :

Theorem ([Hi] $]$ Let $P_{1}, \ldots, P_{m}$ be $m$ polynomials in $\mathbb{C}\left[X_{1}, \ldots, X_{n}\right]$ such that $\operatorname{deg} P_{j} \leq D, j=1, \ldots, m$, and $Q$ lying locally in the integral closure of $\left(P_{1}, \ldots, P_{n}\right)$ at any point in $\mathbb{C}^{n}$ (for example, $Q \in\left(P_{1}, \ldots, P_{m}\right)$ ). Then one can write an explicit division formula:

$$
Q^{\min (n+1, m)}=\sum_{j=1}^{m} P_{j} Q_{j}, \quad \operatorname{deg} P_{j} Q_{j} \leq \min (n+1, m)\left(\operatorname{deg} Q+D^{\min (n, m)}\right) .
$$

A key stumbling block with respect to the use of Briançon-Skoda theorem (or its natural analytic companion, Bochner-Martinelli integral representation formula) when one wants to keep track of algebraic or arithmetic constraints while writing explicit division formulas is the fact that $L^{2}$-methods (or complex analytic methods involving integral representation formulas), usually hide the algebraic argument.

In 1981, J. Lipman and B. Teissier proposed in [LiT] an alternative proof of Briançon Skoda theorem based on the use of multidimensional theory (through integral symbols) : let $f_{1}, \ldots, f_{n}$ define a $\mathfrak{m}$-primary ideal in $\mathcal{O}_{n}$ ( $\mathfrak{m}$ being the maximal ideal in the local ring $\mathcal{O}_{n}$ ) and $h$ lying in the integral closure of $\left(f_{1}, \ldots, f_{n}\right)$; then, for any $g \in \mathcal{O}_{n}$, the Cauchy-like integral

$$
\frac{1}{(2 i \pi)^{n}} \int_{\Gamma_{f}\left(\epsilon_{1}, \ldots, \epsilon_{n}\right)} \frac{h^{n} g d \zeta_{1} \wedge \cdots \wedge d \zeta_{n}}{f_{1} \cdots f_{n}}
$$

where $\Gamma_{f}(\epsilon):=\left\{\left|f_{1}\right|=\epsilon_{1}, \ldots,\left|f_{n}\right|=\epsilon_{n}\right\}$ (which is constant for $\epsilon$ generic thanks to Stokes's theorem) tends to 0 when $\epsilon$ tends to zero (remaining in a cone). Therefore (4) is equal to zero, which implies $h^{n} \in\left(f_{1}, \ldots, f_{n}\right)$ because of the duality theorem for Grothendieck residue symbols attached to regular sequences. The algebraic counterpart of the Cauchy integral symbol is the Cauchy development. It is important to recall here that, for such $f_{1}, \ldots, f_{n}$ and $r \in \mathcal{O}_{n}$, the residue symbol

$$
\operatorname{Res}\left[\begin{array}{c}
r d \zeta_{1} \wedge \cdots \wedge d \zeta_{n} \\
f_{1}, \ldots, f_{n}
\end{array}\right]=\lim _{\epsilon \longrightarrow 0} \frac{1}{(2 i \pi)^{n}} \int_{\Gamma_{f}\left(\epsilon_{1}, \ldots, \epsilon_{n}\right)} \frac{r d \zeta_{1} \wedge \cdots \wedge d \zeta_{n}}{f_{1} \cdots f_{n}}
$$

is not defined alone in algebraic presentations of multidimensional residue theory (as for example in [Lip], chapter 3 ). Such a residue symbol comes as the trace of an operator $T \in \operatorname{Hom}_{\mathbb{C}}\left(\mathcal{O}_{n} /\left(f_{1}, \ldots, f_{n}\right), \mathcal{O}_{n} /\left(f_{1}, \ldots, f_{n}\right)\right)$. It is defined together with the whole list of symbols 


$$
\operatorname{Res}\left[\begin{array}{c}
r d \zeta_{1} \wedge \ldots \wedge d \zeta_{n} \\
f_{1}^{k_{1}+1}, \ldots, f_{n}^{k_{n}+1}
\end{array}\right], \quad\left(k_{1}, \ldots, k_{n}\right) \in \mathbb{N}^{n}
$$

Note that, in the same vein, Briançon-Skoda theorem for $I=\left(f_{1}, \ldots, f_{n}\right)$ is not only the inclusion $\overline{I^{n}} \subset I$, but the whole list of inclusions $\overline{I^{n+k-1}} \subset I^{k}$ for any $k \in \mathbb{N}^{*}$. Here is an alternative way to relate such list of inclusions (in terms of Taylor developments instead of Cauchy integrals) to the duality theorem :

A rephrasing of Briançon-Skoda theorem : Let $\left(f_{1}, \ldots, f_{n}\right)$ and $\left(g_{1}, \ldots, g_{n}\right)$ be two $\mathfrak{m}$-primary ideals in $\mathcal{O}_{n}$ with the same integral closure; then the formal power series (in $\mathbb{C}[[u]]$ )

$$
\sum_{k_{1}=0}^{\infty} \cdots \sum_{k_{n}=0}^{\infty} \operatorname{Res}\left[\begin{array}{c}
r g_{1}^{k_{1}+1} \cdots g_{n}^{k_{n}+1} d \zeta_{1} \wedge \cdots \wedge d \zeta_{n} \\
f_{1}^{k_{1}+1}, \ldots, f_{n}^{k_{n}+1}
\end{array}\right] u_{1}^{k_{1}} \cdots u_{n}^{k_{n}}
$$

is identically zero for any $r \in \mathcal{O}_{n}$.

In order to justify such a reformulation, one should recall here the key remark of $\mathrm{M}$. Hochster in the appendix to $[\mathrm{LiT}]$ : given a regular sequence $\left(f_{1}, \ldots, f_{n}\right)$ in $\mathcal{O}_{n}$, one has, for any $k \in \mathbb{N}^{*}$,

$$
\left(f_{1}, \ldots, f_{n}\right)^{k}=\bigcap_{\substack{l \in \mathbb{N}_{n} \\ l_{1}+\cdots+l_{n}=k-1}}\left(f_{1}^{l_{1}+1}, \ldots, f_{n}^{l_{n}+1}\right) .
$$

Besides the three ways we already mentioned to characterize the membership of a given $h \in \mathcal{O}_{n}$ to the integral closure of some $\mathfrak{m}$-primary ideal $\left(f_{1}, \ldots, f_{n}\right)$, let us formulate here two alternative ones involving multidimensional residue calculus. Both of them appear to be deeply connected with the various techniques we have been developing with Carlos Berenstein (culminating in [BeY4]) in order to make "effective" the black box which corresponds to the involvement of Briançon-Skoda theorem in our work.

Characterization 1 [TsiY], theorem 4.10) An element $h \in \mathcal{O}_{n}$ belongs to the integral closure of $\left(f_{1}, \ldots, f_{n}\right)$ if and only if there exists $N \in \mathbb{N}$ such that for any $r \in \mathcal{O}_{n}$, the power series

$$
\sum_{k_{1}=0}^{\infty} \cdots \sum_{k_{n}=0}^{\infty} \operatorname{Res}\left[\begin{array}{c}
r h^{k_{1}+\cdots+k_{n}} d \zeta_{1} \wedge \cdots \wedge d \zeta_{n} \\
f_{1}^{k_{1}+1}, \ldots, f_{n}^{k_{n}+1}
\end{array}\right] u_{1}^{k_{1}} \cdots u_{n}^{k_{n}}
$$

corresponds to the development about $u=0$ of a rational function $F_{1} / F_{2}$ with no pole at $u=0, F_{2}$ independent of $r$ and $\max \left(\operatorname{deg} F_{1}, \operatorname{deg} F_{2}\right) \leq N$.

Characterization 2 An element $h \in \mathcal{O}_{n}$ belongs to the integral closure of $\left(f_{1}, \ldots, f_{n}\right)$ if and only if there exists $N \in \mathbb{N}$ and $c \in \mathcal{O}_{n}$ such that for any $r \in \mathcal{O}_{n}$, the formal power series

$$
\sum_{k_{1}=0}^{\infty} \cdots \sum_{k_{n}=0}^{\infty} \operatorname{Res}\left[\begin{array}{c}
r c h^{k_{1}+\cdots+k_{n}+1} d \zeta_{1} \wedge \cdots \wedge d \zeta_{n} \\
f_{1}^{k_{1}+1}, \ldots, f_{n}^{k_{n}+1}
\end{array}\right] u_{1}^{k_{1}} \cdots u_{n}^{k_{n}}
$$


is a polynomial in $u$ with degree less than $N$.

Such a characterization is obtained from characterization (3)" using (5) and the duality theorem.

In order to unify the picture (and understand completely the reason why multivariate residue calculus appears as an algebraic counterpart of integral representation formulas or $L^{2}$ methods), it would be crucial to derive a direct "combinatoric" proof of Briançon-Skoda theorem which does not use (even in some artificial way) an integral symbol such as the integration on the chain $\Gamma_{f}(\epsilon)$ in (4). Unfortunately, despite many efforts with M. Hickel, we could not achieve such an objective. Let us sketch briefly what could be a conjectural scheme of the proof : first start with two $\mathfrak{m}$-primary ideals $\left(f_{1}, \ldots, f_{n}\right)$ and $\left(g_{1}, \ldots, g_{n}\right)$ with the same integral closure. Then introducing additional parameters $u_{1}, \ldots, u_{n}$ and noting that formally (this follows from the transformation law in residue calculus, see for example [GrH], chapter 6), one has, for any $r \in \mathcal{O}_{n}$,

$$
\begin{aligned}
& \sum_{k_{1}=0}^{\infty} \cdots \sum_{k_{n}=0}^{\infty} \operatorname{Res}\left[\begin{array}{c}
r g_{1}^{k_{1}+1} \cdots g_{n}^{k_{n}+1} d \zeta_{1} \wedge \cdots \wedge d \zeta_{n} \\
f_{1}^{k_{1}+1}, \ldots, f_{n}^{k_{n}+1}
\end{array}\right] u_{1}^{k_{1}} \cdots u_{n}^{k_{n}} \\
= & \operatorname{Res}\left[\begin{array}{l}
r g_{1} \cdots g_{n} d \zeta_{1} \wedge \cdots \wedge d \zeta_{n} \\
f_{1}-g_{1} u_{1}, \ldots, f_{n}-g_{n} u_{n}
\end{array}\right] \\
= & \frac{(-1)^{n}}{\left(u_{1} \cdots u_{n}\right)^{2}} \operatorname{Res}\left[\begin{array}{c}
r g_{1} \cdots g_{n} d \zeta_{1} \wedge \cdots \wedge d \zeta_{n} \\
g_{1}-f_{1} / u_{1}, \ldots, g_{n}-f_{n} / u_{n}
\end{array}\right]
\end{aligned}
$$

Reversing the roles of $f$ and $g$ and introducing additional parameters $v_{1}, \ldots, v_{n}$ deduce that

$$
\begin{aligned}
& \sum_{k_{1}=0}^{\infty} \cdots \sum_{k_{n}=0}^{\infty} \operatorname{Res}\left[\begin{array}{r}
r f_{1}^{k_{1}+1} \cdots f_{n}^{k_{n}+1} d \zeta_{1} \wedge \cdots \wedge d \zeta_{n} \\
g_{1}^{k_{1}+1}, \ldots, g_{n}^{k_{n}+1}
\end{array}\right] v_{1}^{k_{1}} \cdots v_{n}^{k_{n}} \\
= & \operatorname{Res}\left[\begin{array}{c}
r g_{1} \cdots g_{n} d \zeta_{1} \wedge \cdots \wedge d \zeta_{n} \\
g_{1}-f_{1} v_{1}, \ldots, g_{n}-f_{n} v_{n}
\end{array}\right] .
\end{aligned}
$$

On the other hand, thanks to characterization 2 , the fact that $g_{1}, \ldots, g_{n}$ lie in $\left(f_{1}, \ldots, f_{n}\right)$ implies that there exists a non zero element $c \in \mathcal{O}_{n}$ and $N \in \mathbb{N}$ such that, for any $r \in \mathcal{O}_{n}$, the formal power series

$$
\sum_{k_{1}=0}^{\infty} \cdots \sum_{k_{n}=0}^{\infty} \operatorname{Res}\left[\begin{array}{c}
r c g_{1}^{k_{1}+1} \cdots g_{n}^{k_{n}+1} d \zeta_{1} \wedge \cdots \wedge d \zeta_{n} \\
f_{1}^{k_{1}+1}, \ldots, f_{n}^{k_{n}+1}
\end{array}\right] u_{1}^{k_{1}} \cdots u_{n}^{k_{n}}
$$

is a polynomial in $u$ with degree $N$. Since the polar set of the rational function whose development about the origin is

$$
\sum_{k_{1}=0}^{\infty} \cdots \sum_{k_{n}=0}^{\infty} \operatorname{Res}\left[\begin{array}{c}
r g_{1}^{k_{1}+1} \cdots g_{n}^{k_{n}+1} d \zeta_{1} \wedge \cdots \wedge d \zeta_{n} \\
f_{1}^{k_{1}+1}, \ldots, f_{n}^{k_{n}+1}
\end{array}\right] u_{1}^{k_{1}} \cdots u_{n}^{k_{n}}
$$


does not depend of $r$, there is some hope to show that one get get rid of $c$ and get that such a rational function is in fact a polynomial in $u$, which contradicts (if it is not identically zero) the fact that it could be also expressed as a polynomial in $1 / u_{1}, \ldots, 1 / u_{n}$ (as expected through the transformation law).

Let us go now from the local setting to the global setting and replace the local ring $\mathcal{O}_{n}$ with the polynomial ring $\mathbb{C}\left[X_{1}, \ldots, X_{n}\right]$. Our long term work with Carlos Berenstein convinced me that what makes the algebraic and analytic approaches so different (and therefore so complementary) is that there are two different perceptions of the hyperplane at infinity when dealing with one point of view or the other. In algebraic geometry, the hyperplane at infinity is nothing else than a projective hyperplane (it can be any projective hyperplane after a projective change of coordinates). For the analyst, who looks at algebraic division problems from the affine space $\mathbb{C}^{n}$, the notion of infinity makes sense and carries related notions such as, for example, the notion of topological properness for a polynomial map from $\mathbb{C}^{n}$ to $\mathbb{C}^{m}$, the concept of separation at infinity, together with the related definition of the Lojasiewicz exponent at infinity (see for example [CyKT] for a general overview and references about these fundamental notions which have been extensively studied in the past years by the Crakow school), the concept of apparent contour which was introduced by G. Monge, etc.

Let then $P=\left(P_{1}, \ldots, P_{m}\right)$ be a polynomial map from $\mathbb{C}^{n}$ to $\mathbb{C}^{m}$ such that the zero set $V\left(P_{1}, \ldots, P_{n}\right):=\left\{\zeta \in \mathbb{C}^{n} ; P(\zeta)=0\right\}$ is discrete (hence finite). Let $\mathfrak{I}$ be the ideal generated in $\mathbb{C}\left[X_{0}, \ldots, X_{n}\right]$ by the homogenizations of the $P_{j}$; let $V(\mathfrak{I})$ the zero set of $\mathfrak{I}$ in $\mathbb{P}^{n}(\mathbb{C})$. One can define (see [Hi]) in terms of the polar invariants attached (see [Te1]) to the normalized blow-up of $\mathbb{P}^{n}(\mathbb{C})$ with center $V(\mathfrak{I})$, the least positive rational number $\nu_{\infty}(\mathfrak{I})$ such that

$$
\sum_{j=1}^{m} \frac{P_{j}(\zeta)}{\|\zeta\|^{\operatorname{deg} P_{j}}} \geq \kappa\|\zeta\|^{-\nu_{\infty}(\mathfrak{I})}
$$

for some $\kappa>0$ when $\|\zeta\|>>0$. When $\nu_{\infty}(\mathfrak{I})<\operatorname{deg} P_{j}$ for $j=1, \ldots, m$, the polynomial map $P$ is proper in the topological sense ; the case $\nu_{\infty}(\mathfrak{I})=0$ corresponds to the particular case when the supports of the projective divisors corresponding to $P_{1}, \ldots, P_{m}$ do not intersect on the hyperplane at infinity. The following result (which was transposed in [VY] from the projective setting to the toric setting $[\mathrm{GeKZ}]$ ) appears $[\mathrm{BoH}]$ to be the corner stone of the effective results $[\mathrm{BeY} 2, \mathrm{BeY} 4]$ that gave the first result toward the formulation of an effective arithmetic nullstellensatz :

Theorem ([VY] Assume $\left(P_{1}, \ldots, P_{n}\right)$ is a proper map from $\mathbb{C}^{n}$ to $\mathbb{C}^{n}$. Then, for any $Q \in \mathbb{C}\left[X_{1}, \ldots, X_{n}\right]$ such that

$$
\operatorname{deg} Q<\sum_{j=1}^{n} \operatorname{deg} P_{j}-n\left(1+\nu_{\infty}(\mathfrak{I})\right)=\sum_{j=1}^{n}\left(\operatorname{deg} P_{j}-\nu_{\infty}(\mathfrak{I})\right)-n,
$$


the total residue symbol (that is the sum of local Grothendieck residues at all points in $\left.V\left(P_{1}, \ldots, P_{n}\right)\right)$

$$
\operatorname{Res}\left[\begin{array}{c}
Q(X) d X_{1} \wedge \cdots \wedge d X_{n} \\
P_{1}, \ldots, P_{n}
\end{array}\right]
$$

is equal to 0 .

This result can be proved using Bochner-Martinelli integral representation formula [VY]. M. Hickel noticed that an alternative way to prove it is to use the Briançon-Skoda theorem in its full strength, that is, for any ideal $\mathfrak{I}$ generated by $n$ elements in $\mathcal{O}_{n+1}$ and for any $k \in \mathbb{N}^{*}, \frac{\mathfrak{I}^{k+n-1}}{\mathfrak{I}^{k}}$ for any $k \in \mathbb{N}^{*}$ (note that the assertion for $k=1$ is not sufficient, which shows again that integral symbols involved in Bochner-Martinelli integral formulas hide some Cauchy-like development). One should add here that a currential geometric re-interpretation of this result was recently proposed in [And1].

Because of its ubiquity, Briançon-Skoda theorem provides a dictionary between analytic informations (such as Lojasiewicz inequalities) and algebraic informations (for example the vanishing of a formal power series defined in terms of residue symbols). Here is an example of such a result :

Proposition ([TsiY], lemma 4.2) Let $P_{1}, \ldots, P_{n} \in \mathbb{C}\left[X_{1}, \ldots, X_{n}\right]$ be a proper polynomial map from $\mathbb{C}^{n}$ to $\mathbb{C}^{n}$; the Lojasiewicz exponent at infinity of $P$ is at most 1 (that is $\liminf _{\|\zeta\| \rightarrow \infty}\|P(\zeta)\| /\|\zeta\|^{1-\epsilon}>0$ for any $\epsilon>0$, which is clearly an analytic assertion) if and only if all residue symbols

$$
\operatorname{Res}\left[\begin{array}{c}
r X_{1}^{l_{1}} \cdots X_{n}^{l_{n}} d X_{1} \wedge \cdots \wedge d X_{n} \\
P_{1}^{k_{1}+1}, \ldots, P_{n}^{k_{n}+1}
\end{array}\right], l_{1}+\cdots+l_{n}<k_{1}+\cdots+k_{n}
$$

are equal to zero (this is a list of algebraic relations).

\section{Multiplying integration currents}

At the same time we pushed forward our efforts to clarify the dictionary between analytic tools (such as integral representation formulas) and algebraic ones (multivariate residue calculus adding parameters as described in section 2 ), we went on profiting from analytic aspects of multidimensional residue theory, dealing with the much less rigid currential point of view [Lel]. I will just point out here relevant recent results with potential applications since they are closely related either to integral closure and Briançon-Skoda theorem and to intersection theory. We were interested in both questions with Carlos Berenstein when profiting from the complementarity of analytic, geometric and algebraic ideas. For further references and a complete up-to-date overview of all such topics, I refer to the two survey papers [Bjo] and [TsiY]. 
Let $U$ be some open set in $\mathbb{C}^{n}, F_{1}, \ldots, F_{M} M$ holomorphic functions in $U$. Exploring analytic ideas involved in multivariate residue calculus and inspired (as the analytic ideas lying behind the concept of algebraic closure introduced in the previous section) by normalized blowing-up techniques and toric geometry, we introduced with M. Passare, A. Tsikh and Carlos Berenstein in [Y1, Y2, PTY, BeY4] a list of currents $T_{\mathcal{I}}(F, \mathfrak{p})$ which is indexed by the collection of all non-empty ordered subsets $\mathcal{I}=\left\{i_{1}, \ldots, i_{k}\right\}$ (with $\left.1 \leq i_{1}<\cdots<i_{k} \leq M\right)$ of $\{1, \ldots, M\}$ and by the collection of multi-indexes $\mathfrak{p}=\left(\mathfrak{p}_{1}, \ldots, \mathfrak{p}_{M}\right)$ in $\mathbb{N}^{M}$. For $k \in\{1, \ldots, \min (M, n)\}$ and $\mathcal{I}$ with cardinal $k$, for each $\mathfrak{p} \in \mathbb{N}^{M}$, the "residual current" $T_{\mathcal{I}}(F, \mathfrak{p})$ is the $(0, k)$ current whose action on an $(n, n-k)$-test form $\varphi$ can be expressed as

$$
\left\langle T_{\mathcal{I}}(F, \mathfrak{p}), \varphi\right\rangle:=\lim _{\epsilon \longrightarrow 0} \frac{(-1)^{k(k-1) / 2}(k-1) !}{(2 i \pi)^{k}} \frac{1}{\epsilon^{k}} \int_{\|F\|_{\mathfrak{p}}^{2}=\epsilon} \Omega(F ; \mathcal{I} ; \mathfrak{p}) \wedge \varphi,
$$

where

$$
\begin{aligned}
\|F\|_{\mathfrak{p}}^{2}: & =\sum_{j=1}^{M}\left|F_{j}\right|^{2\left(\mathfrak{p}_{j}+1\right)} \\
\Omega(F ; \mathcal{I} ; \mathfrak{p}): & =\sum_{l=1}^{k}(-1)^{l-1} \overline{F_{i_{l}}} \bigwedge_{\substack{\nu=1 \\
\nu \neq l}}^{k} d\left[\overline{F_{i_{\nu}}}\left|F_{i_{\nu}}\right|^{2 \mathfrak{p}_{i_{\nu}}}\right]
\end{aligned}
$$

The current $T_{\mathcal{I}}(F, \mathfrak{p})$ with $\# \mathcal{I}=k, k=1, \ldots, \min (n, M)$, is annihilated in $U$ (as a current) by holomorphic functions $h$ which locally satisfy about any point in $U$ the condition

$$
\left(\prod_{i \in \mathcal{I}} F_{i}^{\mathfrak{p}_{i}}\right) h \in \overline{\left(F_{1}^{\mathfrak{p}_{1}+1}, \ldots, F_{M}^{\mathfrak{p}_{M}+1}\right)^{\# \mathcal{I}}} .
$$

Moreover the support of all such currents(for any subset $\mathcal{I}$, for any multi-index $\left.\mathfrak{p} \in \mathbb{N}^{n}\right)$ lies in the closed analytic set $V(F)=\{z \in U ; F(z)=0\}$ since it is locally annihilated (as a current) by any anti holomorphic function which vanishes on $V(F)$. From such a property, one could guess, at least heuristically, that the action of the residual current $T_{\mathcal{I}}(F, \mathfrak{p})$ involves only the holomorphic differential operators $\partial / \partial z_{j}$ for $j=1, \ldots, n$. Though they fail to be $\bar{\partial}$-closed (except in the particular case $k=M \leq n$ ), such currents $T_{\mathcal{I}}(F, \mathfrak{p})$ would probably gain to be better understood from the algebraic point of view.

Let $C$ be an effective analytic cycle with support in $U$ and defining ideal (multiplicities being taken into account as in $(2)) I(C)=\left(f_{1}, \ldots, f_{m}\right)$. Let also $S:=\left\{L_{1}=\cdots=L_{r}=0\right\}$ be a $s$ dimensional reduced submanifold in $U$. In $[\mathrm{Tw}]$, P. Tworzewski introduced (through an algorithm inspired by the Vogel-Stückrad construction) the local multi-index of contact $\nu(C, S)=\left(\nu_{s}(C, S), \ldots, \nu_{0}(C, S)\right) \in \mathbb{N}^{s+1}$ between the cycle $C$ and the smooth 
$s$-dimensional submanifold $S$ at some point $x$ lying in the intersection of the support of $C$ and $S$. He also introduced the related intersection cycle $C \bullet S$. It was shown by Achilles and Rams $[\mathrm{AchR}]$ (see also $[\mathrm{AchM}]$ ) that the components of the multi-index of contact $\nu \in \mathbb{N}^{n-r+1}$ can be understood as generalized Hilbert-Samuel multiplicities.

On the other hand, let $M=r+m$ and $F:=\left(L_{1}, \ldots, L_{r}, f_{1}, \ldots, f_{m}\right)$. For any $k \in\{0, s\}$, for any $p \in \mathbb{N}^{r}$, the currents $T_{\mathcal{I}}(F ; \mathfrak{p}$ ), with $\# \mathcal{I}=n-k$ (where $\left.\mathfrak{p}:=\left(p_{1}, \ldots, p_{r}, 0, \ldots, 0\right)\right)$ can be combined with the $d F_{1}, \ldots, d F_{M}$ in order to realize the positive $\partial$ and $\bar{\partial}$-closed $(n-k, n-k)$ current

$$
\left[V_{\mathfrak{p}}(F)\right]_{k}:=\sum_{\substack{\mathcal{I} \\ \# \mathcal{I}=n-k}} \prod_{i \in \mathcal{I}}\left(\mathfrak{p}_{i}+1\right) T_{\mathcal{I}}(F, \mathfrak{p}) \wedge d F_{i_{1}} \wedge \cdots \wedge d F_{i_{n-k}}
$$

(see [BeY5]). When $k=s$, it follows from King's formula [King] that at least if the $p_{j}$ are large enough, the current $\left[V_{\mathfrak{p}}(F)\right]_{s}$ vanishes if $\operatorname{dim}(S \cap$ $\left.V\left(f_{1}, \ldots, f_{m}\right)\right)<s$. Otherwise (see [BeY5, Meo1, Meo2, And3], $S$ is involved as an irreducible component in the decomposition of the analytic subset $S \cap V\left(f_{1}, \ldots, f_{m}\right)$ and the current $\left[V_{\mathfrak{p}}(F)\right]_{s}$ equals $Z_{s}$, where $Z_{s}$ is the $s$ dimensional component of the intersection cycle $C \bullet S$ between $C$ and the smooth manifold $S$ (the local multiplicity at $z$ of such a cycle $Z_{s}$ being the first component in the multi-index of intersection between $C$ and $S$ at the point $z$ in the sense of [Tw]). When $k=0, \ldots, s-1$, the closed positive current $\left[V_{\mathfrak{p}}(F)\right]_{k}$ admits a unique Siu decomposition

$$
\left[V_{\mathfrak{p}}(F)\right]_{k}=\sum_{j} \alpha_{\mathfrak{p}, k, j} C_{\mathfrak{p}, k, j}+R_{\mathfrak{p}, k}
$$

(see [Siu, Dem1, Dem2]) and it seems a natural question to ask whether, for $p_{1}, \ldots, p_{r}$ large enough (or if not for which $p$ ) the singular part

$$
\sum_{j} \alpha_{\mathfrak{p}, k, j} C_{\mathfrak{p}, k, j}
$$

fits with the $k$-dimensional component in the decomposition of the intersection cycle $C \bullet S$ (as defined by Tworzewski in [Tw]). This would give some way to approximate the Tworzewski's multi-index of contact in terms of BochnerMartinelli integral representation formulas (as it of course happens when $C$ and $S$ intersect properly).

Such factorization results of the Lelong-Poincaré type for integration currents in the improper intersection case enhance the role of multidimensional residue theory when multiplying in a robust way (as described in [Bjo]) integration currents with meromorphic forms (either in a product space as for the construction of Green currents [BeY5] or on an incidence manifold as far as the concept of trace is concerned). I will just refer here to our recent work [BeVY] and to the "algebraic" formulations (in terms of the rigidity of a non-linear, 
non-homogeneous system of differential equations whose all solutions are automatically rational when the right-hand side is) of Abel's inverse theorem (see [Gr, HeP, Y3, Wei]). In the same vein I would like also to mention before concluding this section the recent work of B. Fabre [Fab], extending Abel's theorem replacing the notion of trace of a meromorphic form by that of AbelRadon transform of a residual current.

\section{Some conclusive and prospective remarks}

We have seen how simpler the understanding of results such as Briançon-Skoda theorem could be when working in positive characteristic (where the concept of tight closure happens to be a quite useful intermediate notion toward the notion of integral closure). What we tried to point out in this short quite oriented survey is that any time one wants to substitute an algebraic argument to the use of an integral representation, one has to add additional parameters and study the algebraic properties of formal power series involving residual symbols : do such power series correspond to polynomials, developments of rational functions, of algebraic functions, etc. ? The sequence of coefficients of such power series is algebraically governed by transformation laws involved in residue calculus (or generalized versions of transformation laws, as in [BeY4]).

Rigidity constraints that lead to criteria of rationality in $K[[u]]$, where $K$ is a field, imply usually much stronger results in positive characteristic or under arithmetic conditions that allow to use $p$-adic analysis (working over a number field). For example, the combination of Katz and Chudnowsky theorems (as in [DGS, Andr]) is fundamental to conclude to the rationality of solutions of some peculiar differential systems $D-\Gamma$ in $K[[X]]^{\mu}$, where $\Gamma \in \mathcal{M}_{\mu, \mu}(K[X])$, $K$ being a number field.

There seem to be some interest to transpose such ideas (inspired by $p$-adic analysis) to the range of methods we summarized in this very partial presentation. Note that the search for Bernstein-Sato type identities which is central in our attack with Carlos Berenstein of division problems (division being replaced by integration by parts) could also gained to be carried in such a context. This is probably what we missed in our previous study of ideals generated by exponential polynomials. I cannot conclude this survey without mentioning that L. Ehrenpreis's question (are the zeroes of a sum of exponentials with algebraic coefficients and frequencies well separated ?) was (and still remains) a central motivation for introducing all such techniques ; a positive answer to such a question is conditioned by the existence of Bernstein-Sato identities (thinking about analytic division formulas as a tool to attack the problem) or by a better understanding of multidimensional residue calculus (its algebraic companion) in positive characteristic or over a number field (where $p$-adic ideas can be used). 


\section{References}

[AchM] Achilles, R, Manaresi, M. : Multiplicity for ideals of maximal analytic spread and intersection theory, J. Math. Kyoto Univ. 33 (1993), no. 4, pp. 1029-1046.

[AchR] Achilles, R., Rams, S.: Intersection numbers, Segre numbers and generalized Samuel multiplicities, Arch. Math. 77 (2001), pp. 391-398.

[And1] Andersson, M. : Residue currents and ideals of holomorphic functions, Bull. Sci. Math. 128 (2004), no. 6, pp. 481-512.

[And2] Andersson, M. : The membership problem for polynomial ideals in terms of residue currents, preprint (2004), 15 pages.

[And3] Andersson, M. : Residues of holomorphic sections and Lelong currents, to appear in Arkiv for matematik, 16 pages.

[Andr] André, Y. : Séries Gevrey de type arithmétique, II. Transcendance sans transcendance, Annals of Math. 151 (2000), pp. 741-756.

[BeGVY] Berenstein, C.A., Gay, R., Vidras, A., Yger, A.: Residue currents and Bezout identities, Progress in Math. 114, Birkhäuser, 1993.

[BeY1] Berenstein, C.A., Yger, A.: Ideals generated by exponential-polynomials, Advances in Math. 60 (1986), pp. 69-120.

[BeY2] Berenstein, C.A., Yger, A.: Effective Bézout identitities in $\mathbb{Q}\left[z_{1}, \ldots, z_{n}\right]$, Acta Math. 166 (1991), pp. 69-120.

[BeY3] Berenstein, C.A, Yger, A.: Formules de représentation intégrale et problèmes de division, in Diophantine Approximations and Transcendental Numbers, Luminy 1990, P. Philippon Ed., Walter de Gruyter, New York 1992, pp. 15-37.

[BeY4] Berenstein, C.A., Yger, A.: Residue Calculus and effective Nullstellensatz, American Journal of Mathematics, 121 (1999), no. 4, pp. 723-796.

[BeY5] Berenstein, C.A., Yger, A.: Residue currents, integration currents in the non complete intersection case, J. reine. angew. Math. 527 (2000), pp. 203-235.

[BeVY] Berenstein, C.A., Vidras, A., Yger, A.: Analytic residues along algebraic cycles, Journal of Complexity, 21 (2005), no. 1, pp. 5-42.

[Bjo] Björk, J.E.: Residues and D-modules, dans The Legacy of Niels Henrik Abel, the Abel Bicentennial, Oslo 2002 ; Laudal, Olav Arnfinn; Piene, Ragni (Eds.), Springer-Verlag, 2004, pp. 605-652.

[BoGS] Bost, J.-B., Gillet, H., Soulé, C.: Heights of projective varieties and positive Green forms, J. Amer. Math. Soc. 7 (1994), pp. 903-1027.

[BoH] Boyer, J.Y., Hickel, M.: Extension dans un cadre algébrique d'une formule de Weil, manuscripta math. 98 (1999), pp. 1-29.

[BriS] Briançon, J., Skoda, H.: Sur la clôture intégrale d'un idéal de germes de fonctions holomorphes en un point de $\mathbb{C}^{n}$, Comptes Rendus Acad. Sci. Paris, série A, 278 (1974), pp. 949-951.

[Bro] Brownawell, D.W.: Bounds for the degrees in the Nullstellensatz, Ann. of Math. 126 (1987), pp. 577-592.

[CyKT] Cygan, E., Krasiński, T., Tworzewski, P., Separation of algebraic sets and the Lojasiewicz exponent of a polynomial mapping, Invent. math. 136 (1999), no. 1, pp. $75-87$.

[Dem1] Demailly, J.-P.: Courants positifs et théorie de l'intersection, Gaz. Math. 53 (1992), pp. 131-159.

[Dem2] Demailly, J.-P.: Monge-Ampère operators, Lelong numbers and intersection theory, in Complex analysis and geometry, Univ. Ser. Math., Plenum, New York, 1993, pp. 115-193. 
[DGS] Dwork, B., Gerotto, G., Sullivan, F.J : An introduction to G-functions, Annals of Mathematics Studies, 133, Princeton University Press, Princeton, NJ, 1994. xxii+323 pp.

[EL] Ein, L., Lazarsfeld, R., A geometric effective nullstellensatz, Invent. Math. 137 (1999), pp. 427-448.

[Fab] Fabre, B: Sur la transformation d'Abel-Radon des courants localement résiduels, C. R. Math. Acad. Sci. Paris 338(2004), no. 10, 787-792.

[GeKZ] Gelfand I.M., Kapranov M.M, Zelevinsky, A.V, Discriminants, Resultants and Multidimensional Determinants, Birkhäuser, Boston, 1994.

[Gr] Griffiths, P.: Variations on a theorem of Abel, Invent. math. 35 (1976), pp. 321-390.

[GrH] Griffiths, P., Harris, J.: Principles of algebraic geometry, Wiley Interscience, New York, 1978.

[HanR] Hanner, O., and Radström, H. : A generalization of a theorem of Fenchel, Proc. A.M.S. 2 (1951), pp. 589-598.

[Har] Harvey, R.: Integral formulae connected by Dolbeault's isomorphism, Rice University Studies 56 1970, no. 2 (1971), pp. 77-97.

[HeP] Henkin, G., Passare, M.: Abelian differentials on singular varieties and variation on a theorem of Lie-Griffiths, Invent. Math. 135 (1999), pp. 297-328.

[Hi] Hickel, M.: Solution d'une conjecture de C. Berenstein-A. Yger et invariants de contact à l'infini, Ann. Inst. Fourier, Grenoble, 51 (2001), no. 3, pp. 707-744.

[HoH1] Hochster, M., Huneke, C.: Tight closure, invariant theory, and the BriançonSkoda theorem, Jour. Amer. Math. Soc. 3 (1990), pp. 31-116.

[HoH2] Hochster, M., Huneke, C.: Tight closure in equal characteristic zero, preprint, 204 pages.

[King] King, J.: A residue formula for complex subvarieties, in Proc. Carolina conf. on holomorphic mappings and minimal surfaces, Univ. of North Carolina, Chapel Hill, 1970, pp. 43-56.

[Ko1] Kollár, J.: Sharp effective nullstellensatz, J. Amer. Math. Soc. 1 (1988), pp. 963-975.

[Ko2] Kollár, J.: Effective Nullstellensatz for arbitrary ideals, J. Eur. Math. Soc. 1 (1999), pp. 313-337.

[KPS] Krick, T., Pardo, L.M., Sombra, M.: Sharp estimates for the arithmetic Nullstellensatz, Duke Math. J. 109 (2001), no. 3, pp. 521-598.

[LejT] Lejeune-Jalabert, M., Teissier, B.: Clôture intégrale des idéaux et équisingularité, Séminaire Lejeune-Teissier 1974, Publ. Institut Fourier, Grenoble.

[Lel] Lelong, P.: Fonctions plurisousharmoniques et formes différentielles positives, Gordon and Breach science publishers, 1968.

[Ler] Leray, J.: Le calcul différentiel et intégral sur une variété analytique complexe. (Problème de Cauchy. III), Bull. Soc. Math. France 87 (1959), pp. 81-180.

[Lip] Lipman, J.: Residues and traces of differential forms via Hochschild homology, Contemporary Mathematics 61, American Mathematical Society, Providence, 1987.

[LiS] Lipman, J., Sathaye, A.: Jacobian ideals and a theorem of Briançon-Skoda, Michigan Math J. 28 (1981) pp. 199-222.

[LiT] Lipman, J., Teissier, B.: Pseudo-rational local rings and a theorem of Briançon-Skoda about integral closures of ideals, Michigan Math. J. 28 (1981), pp. $97-116$.

[Meo1] Méo, M.: Résidus dans le cas non nécessairement intersection complète, Comptes Rendus Acad. Sci. Paris Sér. I Math. 333 (2001), no. 1, pp. 33-38. 
[Meo2] Méo, M.: Courants résidus et formule de King, preprint.

[Noe] Nöther, M.: Ueber einen Satz aus der Theorie der algebraischen Functionen, Math. Ann. 6 (1873), no. 1, pp. 351-359.

[Nor] Norguet, F.: Dérivées partielles et résidus de formes différentielles sur une variété analytique complexe, Séminaire Lelong, 1958/59, exposé 10.

[PTY] Passare, M., Tsikh, A., Yger, A.: Residue currents of the Bochner-Martinelli type, Publicacions Matematiques 44 (2000), pp. 85-117.

[Siu] Siu, Y.T.: Analyticity of sets associated to Lelong numbers and the extension of closed positive currents, Invent. math. 27 (1974), pp. 53-156.

[Smi] Smith, K.E., An introduction to tight closure, in Geometric and combinatorial aspects of commutative algebra, Messina 1999, Lecture Notes in Pure and Appl. Math. 217, Dekker, New York, 2001, pp. 353-377.

[Te1] Teissier, B.: Variétés polaires II, in Algebraic Geometry, La Rabida, Lecture Notes in Mathematics 961, Springer-Verlag, 1980, pp. 71-146.

[Te2] Teissier, B.: Résultats récents d'algèbre commutative effective, in Séminaire Bourbaki 1989-1990, Astérisque 189-190, Soc. Math. France, pp. 107-131.

[Te3] Teissier, B.: Monomial ideals, binomial ideals, polynomial ideals, in Proceedings of the Introductory Workshop in Commutative Algebra, MSRI, September 2002, to appear.

[TsiY] Tsikh, A., Yger, A.: Residue currents, Journal of Mathematical Sciences 120 (2004), no. 6, pp. 1916-1971.

[Tw] P. Tworzewski: Intersection theory in complex analytic geometry, Ann. Polon. Math. 62 (1995), pp. 177-191.

[VY] Vidras, A., Yger, A.: On some generalizations of Jacobi's residue formula, Annales Scien. École Norm. Sup. 34 (2001), pp. 131-157.

[We] Weil, A.: L'intégrale de Cauchy et les fonctions de plusieurs variables, Math. Ann. 111 (1935), pp. 178-182.

[Wei] Weimann, Martin: La trace via le calcul résiduel : une nouvelle version du théorème d'Abel inverse ; formes abéliennes, preprint math.CV/0405591, 33 pages.

[Y1] Yger, A., Résidus, courants résiduels et courants de Green, in Géométrie complexe, Actualités Sci. Indust., 1438, Hermann, Paris, 1996, pp. 123-147.

[Y2] Yger, A., Aspects opérationnels de la théorie des résidus hors du cadre intersection complète, in Géométrie Complexe II, Hermann, Paris, 2004, pp. 182-204.

[Y3] Yger, A.: Abel's theorem revisited through new developments in residue theory and effectivity, preprint, 19 pages. 